\title{
Probabilistic Radiographic Atlas of Glioblastoma Phenotypes
}

\author{
B.M. Ellingson, A. Lai, R.J. Harris, J.M. Selfridge, W.H. Yong, K. Das, W.B. Pope, P.L. Nghiemphu, H.V. Vinters,
} L.M. Liau, P.S. Mischel, and T.F. Cloughesy

\begin{abstract}
BACKGROUND AND PURPOSE: Tumor location is a significant prognostic factor in glioblastoma, which may reflect the genetic profile of tumor precursor cells. The purpose of the current study was to construct and analyze probabilistic radiographic atlases reflecting preoperative tumor locations and corresponding demographic, "-omic," and interventional phenotypes to provide insight into potential niche locations of glioblastoma cells of origin.
\end{abstract}

MATERIALS AND METHODS: Preoperative anatomic MR images in 507 patients with de novo glioblastoma were analyzed. Images were registered to stereotactic space, tumors were segmented, and the stereospecific frequency of tumor occurrence was analyzed statistically by age, extent of resection, MGMT methylation, IDH1 mutation, gene expression subclassification, PTEN loss, PTEN deficiency, EGFR amplification, EGFR variant 3 expression, progression-free survival from the start of radiochemotherapy, and overall survival from initial diagnosis.

RESULTS: Most glioblastomas grow into the periventricular white matter regions adjacent to the subventricular zone. MGMT promoter methylated tumors occur more frequently in the left temporal lobe, in young patients with glioblastoma, in IDHI mutant tumors, in tumors having the proneural gene expression subtype, and in tumors lacking loss of PTEN occurring most frequently in the frontal lobe. MGMT methylated tumors with the IDHI mutation tended to occur in the left frontal lobe. EGFR amplified and EGFR variant 3-expressing tumors occurred most frequently in the left temporal lobe. A similar region in the left temporal lobe was associated with favorable response to radiochemotherapy and increased survival.

CONCLUSIONS: Radiographic atlases for specific phenotypes provide insight into overlap between prognostic variables and may help to identify niche locations for cancer cells of origin.

ABBREVIATIONS: ADIFFI = analysis of differential involvement; EGFR = epidermal growth factor receptor; IDHI = isocitrate dehydrogenase 1; MGMT = O6methylguanine methyltransferase; PTEN $=$ phosphatase and tensin homolog; $\mathrm{T}+\mathrm{C}=$ postcontrast T1-weighted image

dentifying brain tumor precursor cells and their “-omic" signatures is a major challenge in the field of neuro-oncology that holds tremendous promise for increasing survival by customizing

\section{Received May 20, 2012; accepted after revision May 26.}

From the Departments of Radiological Sciences (B.M.E., R.J.H., W.B.P.), Biomedical Physics (B.M.E., R.J.H.), Biomedical Engineering (B.M.E.), Neurology (A.L., J.M.S., P.L.N., T.F.C.), Pathology and Laboratory Medicine (W.H.Y., K.D., H.V.V., P.S.M.), and Neurosurgery (L.M.L.), David Geffen School of Medicine, University of California, Los Angeles, Los Angeles, California.

This work was supported by the University of California Cancer Research Coordinating Committee Grant (B.M.E.); a UCLA Radiology Exploratory Research Grant (B.M.E.); a UCLA Institute for Molecular Medicine Seed Grant (B.M.E.); the Art of the Brain (T.F.C.); the Ziering Family Foundation in memory of Sigi Ziering (T.F.C.); the Singleton Family Foundation (T.F.C.); and the Clarence Klein Fund for NeuroOncology (T.F.C.).

Please address correspondence to Benjamin M. Ellingson, PhD, Department of Radiological Sciences, David Geffen School of Medicine, University of California, Los Angeles, 924 Westwood Blvd, Suite 615, Los Angeles, CA 90024; e-mail: bellingson@mednet.ucla.edu

http://dx.doi.org/10.3174/ajnr.A3253 treatment paradigms. Evidence suggests that tumor location plays an important role in prognosis ${ }^{1}$ and is likely linked to the genetic profile of tumor cells of origin. ${ }^{2,3}$ In support of this hypothesis, region-specific brain tumor cells of origin have been identified in oligodendrogliomas, ${ }^{4}$ medulloblastomas, ${ }^{5}$ and ependymomas, ${ }^{6}$ and compelling evidence has suggested that this is the case for IDH1 mutated gliomas. ${ }^{7}$ Further, studies have identified clear links between radiologic features, including the volume and extent of edema and/or invasion ${ }^{8}$ as well as contrast enhancement, ${ }^{9}$ and -omic signatures. On the basis of these findings, we have constructed a set of radiographic atlases specifying the probability of tumor location and volumetric information for important demographic, -omic, and interventional phenotypes, with the goal of providing new insight into the possible niche locations of cells of origin in glioblastoma. The radiographic atlases of glioblastoma phenotypes presented in the current study quantify the probability of tumor occurrence in 507 glioblastomas stratified by 
age, extent of resection, MGMT promoter methylation, IDH1 mutation, gene expression subclassification, PTEN loss, PTEN deficiency, EGFR amplification, EGFR variant 3 expression, time to progression from the start of radiochemotherapy, and overall survival from initial diagnosis, along with some interactions between these phenotypes.

\section{MATERIALS AND METHODS Patients}

Five hundred seven patients with de novo histologically confirmed glioblastoma between April 2000 and December 2011 were enrolled in the current retrospective, institutional review boardapproved, Health Insurance Portability and Accountability Actcompliant study. All patients had presurgical T2/FLAIR and postcontrast T1-weighted images available. Subsets of these patients were used in other studies. ${ }^{7,10,11}$ The extent of resection was noted for the initial surgery. Overall survival was defined as the time from initial pathologic diagnosis until death.

\section{MR Imaging}

Data were collected on either a 1.5T or 3T MR imaging scanner. Standard anatomic MR imaging sequences consisted of T2weighted FSE or FLAIR images, and gadolinium-diethylene-triamine pentaacetic acid- (Magnevist; Berlex, Wayne, New Jersey; $0.1 \mathrm{mmol} / \mathrm{kg}$ ) or gadobenate dimeglumine- (MultiHance; Bracco, Milan, Italy; $0.1 \mathrm{mmol} / \mathrm{kg}$ ) enhanced axial $\mathrm{T} 1-$ weighted images $(\mathrm{ie}, \mathrm{T} 1+\mathrm{C})$. All images were 3- to 5-mm thick with a 0 - to $1-\mathrm{mm}$ intersection gap.

\section{Image Registration}

All images for each patient were registered to a $1.0-\mathrm{mm}$ isotropic brain atlas (Montreal Neurological Institute 152) by using a mutual information algorithm and a $12 d f$ transformation by using the Functional MR Imaging of the Brain Software Library (http:// www.fmrib.ox.ac.uk/fsl/) followed by visual inspection and a consensus by 2 independent raters (W.B.P. and B.M.E.) to ensure adequate alignment.

\section{ROIs}

After image registration, T2/FLAIR and T1+C images were segmented by using a semiautomated procedure previously documented. ${ }^{10,12}$ Briefly, the general regions of tumor on T2/FLAIR and $\mathrm{T} 1+\mathrm{C}$ images were first defined manually. Then, T2/FLAIR and $\mathrm{T} 1+\mathrm{C}$ images were thresholded by using an empiric threshold combined with a region-growing algorithm confined to the manually defined ROIs in the first step. Last, tumor regions were manually edited to exclude any obvious errors in segmentation. Regions of central necrosis were included in the study to outline the entire extent of the tumor. Tumor volume was calculated with respect to preregistration image resolution.

\section{ADIFFI}

ADIFFI analysis consisted of first constructing a $2 \times 2$ contingency table comparing 2 differential phenotypes (eg, phenotypes A and B) and tumor versus nontumor for each image voxel. Next, a 2-tailed Fisher exact test was performed on a voxelwise basis.
According to the Fisher exact test, the probability of obtaining an observed pattern in the $2 \times 2$ contingency table is given by

1)

$$
p=\frac{(a+b) !(c+d) !(a+c) !(b+d) !}{a ! b ! c ! d ! n !}
$$

where $a$ is the frequency of tumor occurrence in a particular voxel for phenotype $\mathrm{A} ; b$, the frequency of tumor occurrence in a particular voxel for phenotype $B$; $c$, the frequency of no tumor occurring in a particular voxel for patients with phenotype $\mathrm{A}$; $d$, the frequency of no tumor occurring in a particular voxel for patients with phenotype $\mathrm{B} ; n$, the total number of patients included; and the exclamation point represents the factorial operation. To calculate the significance of the observed pattern in the contingency table corresponding to the total probability of observing a pattern in the contingency table as extreme or more extreme, we recalculated the $P$ value from each voxel for all cases in which the marginal totals were the same as the observed tables and only for cases in which the arrangement was as extreme as the observed pattern. We performed this iteratively so that the values were incremented to calculate a more extreme pattern, adding the previous $P$ value in each image voxel each time until the most extreme pattern was achieved (which may vary from voxel to voxel). The final $P$ value represents the probability of observing the given pattern in the contingency table by chance. $P<.05$ was considered significant. Additional details are presented in a previous publication. ${ }^{10}$

\section{Correction for Cluster Size by Using Random Permutations}

A cluster-based permutation correction was performed as outlined by Bullmore et al. ${ }^{13} \mathrm{~A}$ total of 500 permutations were performed, the resulting ADIFFI-defined clusters were retained, and the 95\% confidence intervals for significant cluster size occurring by chance were documented for each phenotype. The cluster-size thresholds used for each phenotype had a $<5 \%$ probability of occurring by chance.

\section{Molecular and Genetic Analysis}

MGMT methylation analysis was performed by methylation-specific polymerase chain reaction or real-time methylation-specific polymerase chain reaction (LabCorp, Santa Monica, California), according to previously published protocols. ${ }^{10,14,15} \mathrm{IDH} 1$ mutation status was determined by subjecting DNA to amplification by polymerase chain reaction by using primers specific for the IDHI gene, followed by DNA sequencing analysis of a region surrounding codon 132 as described in Lai et al. ${ }^{7}$ Gene-expression subclassification was performed according to standard protocols and categories. ${ }^{16}$ EGFR amplification was performed by the UCLA Cytogenetics Laboratory (Los Angeles, California) by using fluorescence in situ hybridization (Abbott/Vysis, Des Plaines, Illinois; LSI EGFR/CEP7 probe cocktail specific for the 7p12 EGFR locus and centromere of chromosome 7). The PTEN copy number was analyzed by using fluorescence in situ hybridization (Abbott/Vysis; LSI PTEN/CEP10 probe cocktail specific for the 10q23 PTEN locus and centromere of chromosome 10). PTEN deficiency was determined by immunohistochemistry (Sarkaria Biomarker Innovations Laboratory or Brain Tumor Translational Resource at UCLA, Los Angeles, California) by using standard protocols. ${ }^{17}$ Tissue sections were stained with monoclonal antibodies for 


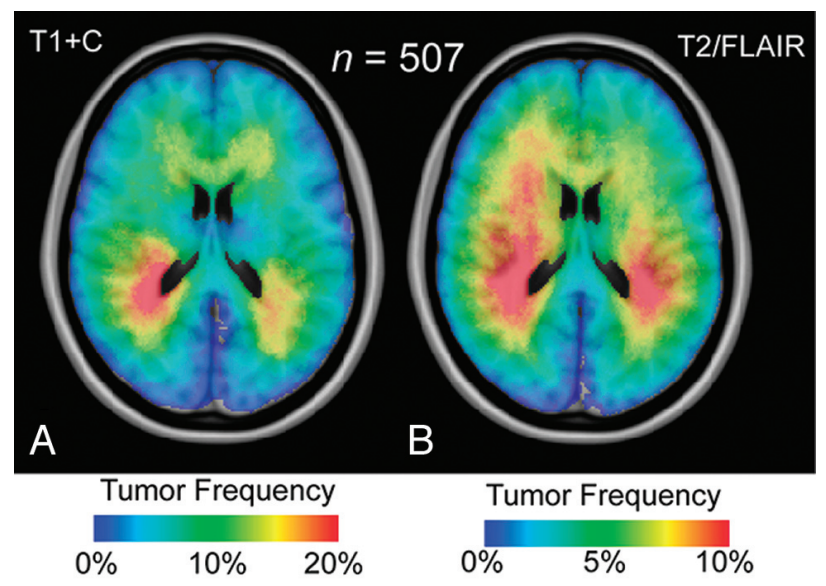

FIG 1. Atlas of all glioblastoma locations. Frequency of glioblastoma occurrence for contrast-enhancing $(T 1+C)(A)$ and T2/FLAIR $(B)$ hyperintense lesions in 507 patients. Regions with the highest tumor frequency occur in the periventricular white matter regions adjacent to the subventricular zone.

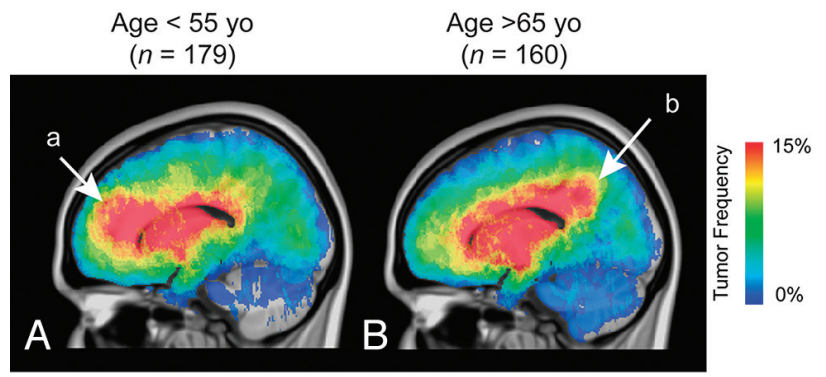

FIG 2. Atlas of glioblastoma location by patient age. Stratification of glioblastoma tumor location by age shows a high incidence in the frontal lobe in younger patients ( $a$ in $A$ ) in contrast to the high incidence in more posterior regions in older patients ( $b$ in $B$ ). T2/FLAIR tumor frequency is illustrated.

PTEN (clone 6H2.1; Cascade Bioscience, Winchester, Massachusetts). EGFR variant 3 expression was determined by immunohistochemistry ${ }^{17}$ or real-time polymerase chain reaction. ${ }^{18}$

\section{Time to Progression for Radiochemotherapy Interventional Phenotype}

Patients treated with upfront radiochemotherapy (radiation therapy and concomitant TMZ temozolomide followed by adjuvant TMZ temozolomide as described by Stupp et $\mathrm{al}^{19}$ ) showing progression before the last date of censorship $(n=374)$ were stratified on the basis of the time to treatment failure. Centralized review of progression-free survival was performed for all patients with available scans as previously described. ${ }^{7,10}$ The date of death was used as the data of progression if the patient had a stable scan finding $<2$ months before death.

\section{RESULTS}

A substantial number of glioblastomas grew into the periventricular white matter regions adjacent to the subventricular zone (Fig $1 A,-B$ ), and $91.9 \%$ of glioblastomas had T2/FLAIR signal abnormality contiguous with the ventricular system. Tumor frequency results stratified by age suggested frontal lobe predominance in younger patients ( $\mathrm{a}$ in Fig $2 A$ ) compared with older patients (b in Fig $2 B$ ). ADIFFI statistical analysis performed on glioblastomas

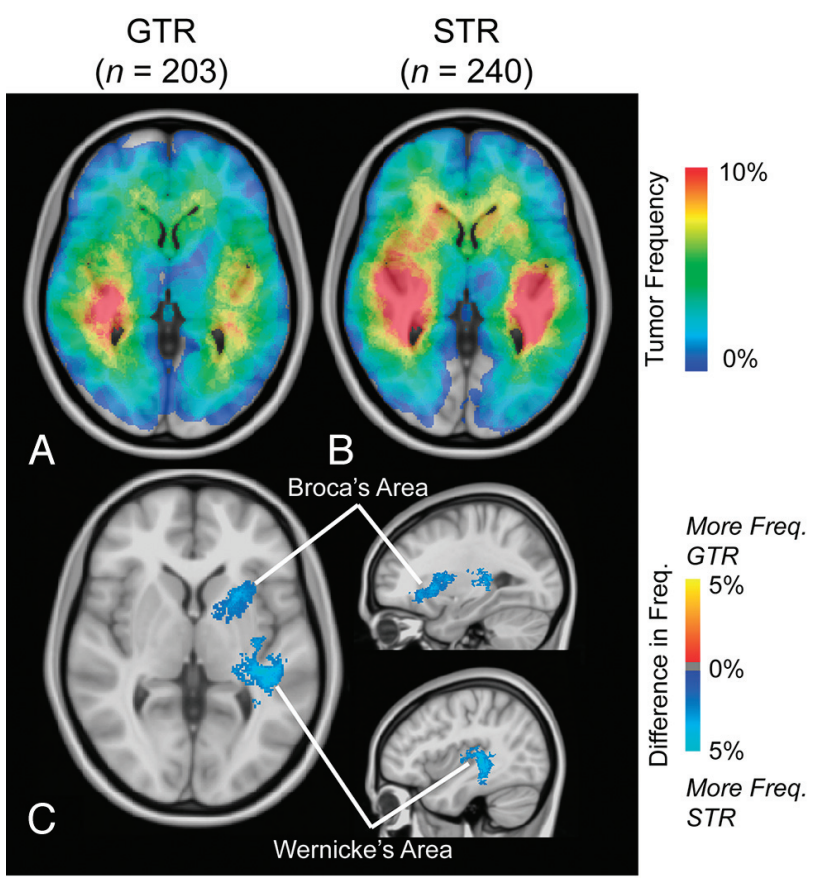

FIG 3. Atlas of glioblastoma location for different extents of resection. Frequency of $\mathrm{Tl}+\mathrm{C}$ tumor occurrence for gross total resection (GTR, $n=203$ ) (A) and subtotal resection (STR, $n=240)(B)$. C, ADIFFI statistical analysis illustrates a single significant cluster (contiguous, out of plane) occurring at a high frequency in patients with STR, encompassing both Broca and Wernicke areas. $\mathrm{Tl}+\mathrm{C}$ tumor frequency is illustrated.

stratified by the extent of resection (gross total resection versus subtotal resection) identified a single spatially distinct cluster encompassing both Broca and Wernicke areas that was statistically more likely to be subtotal resection (Fig 3).

\section{MGMT Promoter Methylation and IDH1 Mutation}

Four hundred thirty-three patients had MGMT promoter methylation data available, for which $38 \%$ had MGMT promoter methylation. A subset of patients was also included from a previous study investigating MGMT promoter methylation. ${ }^{10}$ ADIFFI analysis of glioblastomas based on MGMT promoter methylation status identified a cluster in the right temporal lobe as most frequently associated with MGMT promoter unmethylated tumors and a similar cluster in the left temporal lobe most frequently associated with MGMT promoter methylated tumors (a in Fig $4 A$ ), similar to previous data. ${ }^{10}$ MGMT promoter methylated tumors had a lower volume of contrast enhancement (Fig $4 C$; $t$ test, $P=.038$ ) and T2/FLAIR hyperintensity (Fig $4 C$; $t$ test, $P=.004$ ) compared with unmethylated tumors. Four hundred patients with glioblastoma had IDH1 mutation status information available, for which $8.5 \%$ of glioblastomas were IDH1-mutated. A spatially distinct region in the frontal lobe occurred at a significantly higher frequency in IDH1-mutant tumors (Fig 4B), similar to previously presented data. ${ }^{7}$ IDH1 wild-type glioblastomas had larger volumes of contrast enhancement (Fig $5 B$; $t$ test, $P=.005$ ) but no difference in T2/FLAIR volume (Fig $5 B$; $t$ test, $P=.645$ ). ADIFFI statistical analysis of the interaction between MGMT and IDH1 phenotypes identified 2 independent clusters when IDH1 mutation status was compared within MGMT promoter methyl- 


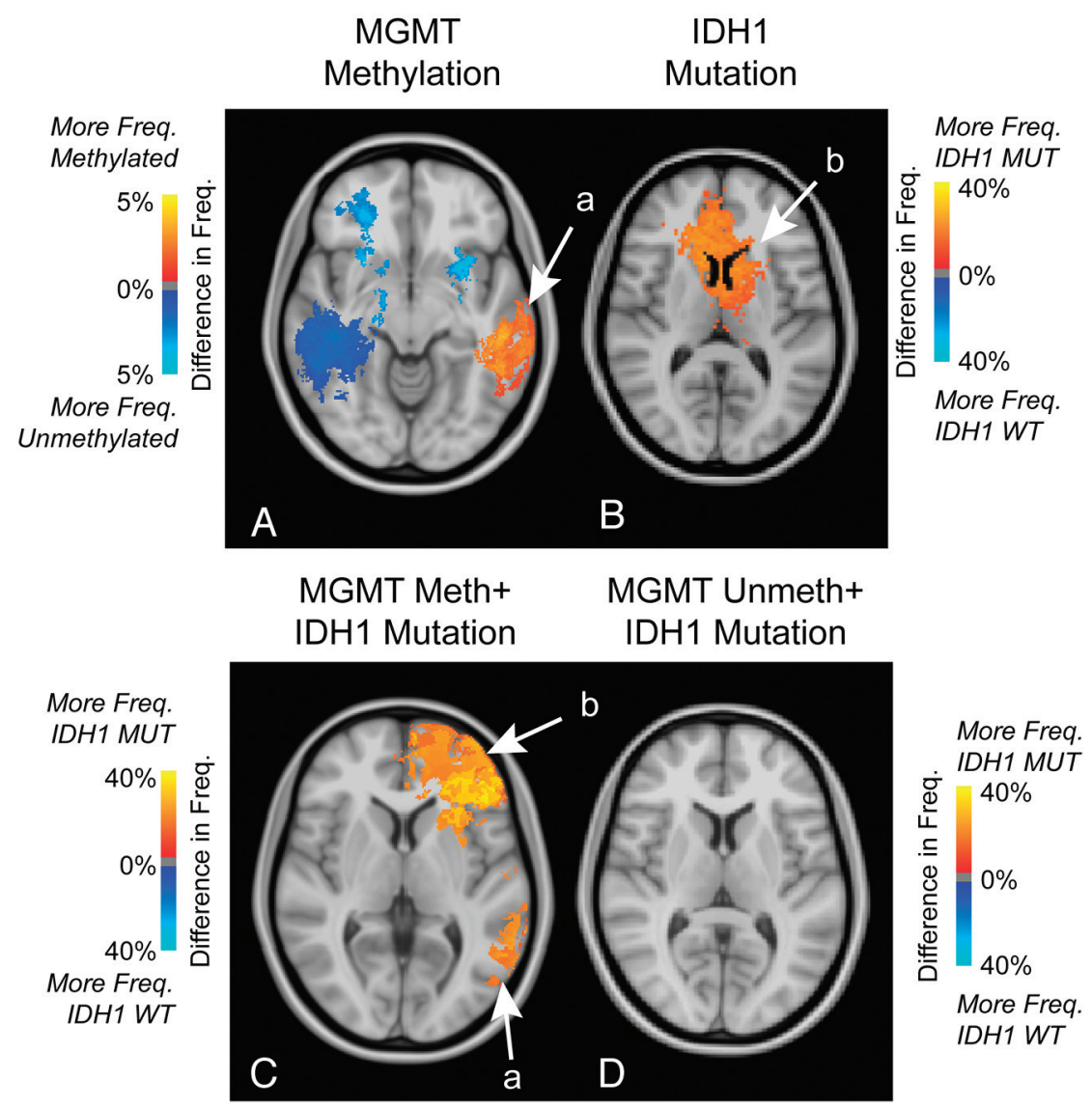

FIG 4. Atlases of MGMT promoter methylation, IDH1 mutation, and the interaction between MGMT methylation and IDHI mutation. A, ADIFFI statistical analysis for MGMT methylation status illustrating cluster a in the left temporal lobe corresponding to regions with a significantly higher frequency of MGMT promoter methylated tumors (methylated [ $n=269]$ and unmethylated $[n=164]$ ). $B$, ADIFFI analysis for IDHI mutation status illustrating a significant cluster $b$ in the frontal lobe, corresponding to regions with a significantly higher frequency of IDHI mutant (MUT) tumors (IDHI MUT [ $n=34]$ and IDHI wild-type [WT] [ $n=366])$. C, ADIFFI statistical analysis for IDHI mutation status within MGMT promoter methylated tumors illustrates both cluster $a$ in the temporal lobe and cluster $b$ in the frontal lobe as having a significantly higher frequency of IDHI mutant tumors (MGMT methylated + IDHI MUT [ $n=15]$ and MGMT methylated + IDHI WT $[n=139]$ ). D, ADIFFI statistical analysis for IDHI mutation status within MGMT promoter unmethylated tumors did not show any significant difference between topographic location of IDHI MUT and WT tumors (MGMT unmethylated + IDHI MUT [ $n=10]$ and MGMT unmethylated + IDHI WT [ $n=217])$. FLAIR tumor frequency is illustrated.

associated with a significantly higher likelihood of lacking PTEN loss (Fig 6A). No differences in lesion volumes were found between tumors with PTEN loss and those without loss of PTEN ( $t$ test, $P>$ .05). ADIFFI analysis of PTEN protein deficiency identified a single contiguous cluster having a significantly higher likelihood of being PTEN deficient running along the posterior aspect of the left lateral ventricle (Fig 6B). No differences in lesion volumes were found between $P T E N$-deficient and intact tumors ( $t$ test, $P>.05)$.

\section{EGFR Amplification and EGFR variant 3 Expression}

EGFR amplification information was available on 136 patients, of which $42 \%$ showed amplification. EGFR variant 3 expression was observed in $25 \%$ of the 48 patients with data available. ADIFFI analysis isolated 2 clusters both containing a significantly higher proportion of tumors exhibiting EGFR amplification (Fig 7A). ADIFFI analysis of EGFR variant 3 expression also identified 1 of the 2 clusters (a in Fig 7B). EGFR-amplified tumors had a significantly higher $\mathrm{T} 1+\mathrm{C}$ ( $t$ test, $P=$ .049) and T2/FLAIR hyperintense volume ( $t$ test, $P=.032$ ) compared with tumors lacking EGFR amplification. No difference in tumor volumes was detected between EGFR variant 3-expressing tumors and tumors lacking EGFR variant 3 expression ( $t$ test, $P>.05$ ).

\section{Radiochemotherapy Interventional Phenotypes}

Three hundred seventy-four patients with glioblastomas were initially treated with

ated tumors in similar locations with the independently analyzed MGMT and IDH1 phenotypes (Fig 4C). No statistically significant clusters were identified when comparing $I D H 1$ mutation status within MGMT unmethylated tumors (Fig 4D).

\section{Gene-Expression Subtypes}

Topographic comparisons with respect to gene-expression subtypes revealed strong frontal lobe predominance in the proneural + proliferative genetic subtype (Fig $5 B$ ). The mesenchymal subgroup appeared to have the highest frequency of occurrence in diffuse regions throughout the right hemisphere (Fig 5A). ADIFFI statistical analysis confirmed independent localization of these 2 gene-expression phenotypes ( $\mathrm{a}$ and $\mathrm{b}$ in Fig $5 C$ ).

\section{PTEN Loss and PTEN Deficiency}

PTEN loss was apparent in $86 \%$ of the 95 patients with data available. ADIFFI analysis identified a cluster in the left frontal lobe radiochemotherapy and showed progression at the time of censorship. ADIFFI analysis of tumor location between nonresponders $(<6$-month time to progression) and long-term responders ( $>12$-month time to progression) identified 2 clusters ( $\mathrm{a}$ and $\mathrm{b}$ in Fig $8 C$ ), both associated with a favorable response. No relationships between tumor volume and time to progression were identified (T1+C and T2/FLAIR, 1-way ANOVA; <6month time to progression, 6- to 12-month time to progression, and $>12$-month time to progression; $P>.05$ ). When examining the interaction between MGMT promoter methylation and time to progression, ADIFFI statistical analysis identified 2 distinct clusters ( $\mathrm{a}$ and $\mathrm{b}$ in Fig $8 D$ ) in a similar location in all glioblastomas; however, the lateral portion of cluster a and the anterolateral portion of cluster $b$ were associated with long-term responders, whereas medial regions in cluster a and posterior regions in cluster b were regions associated with nonresponders. 


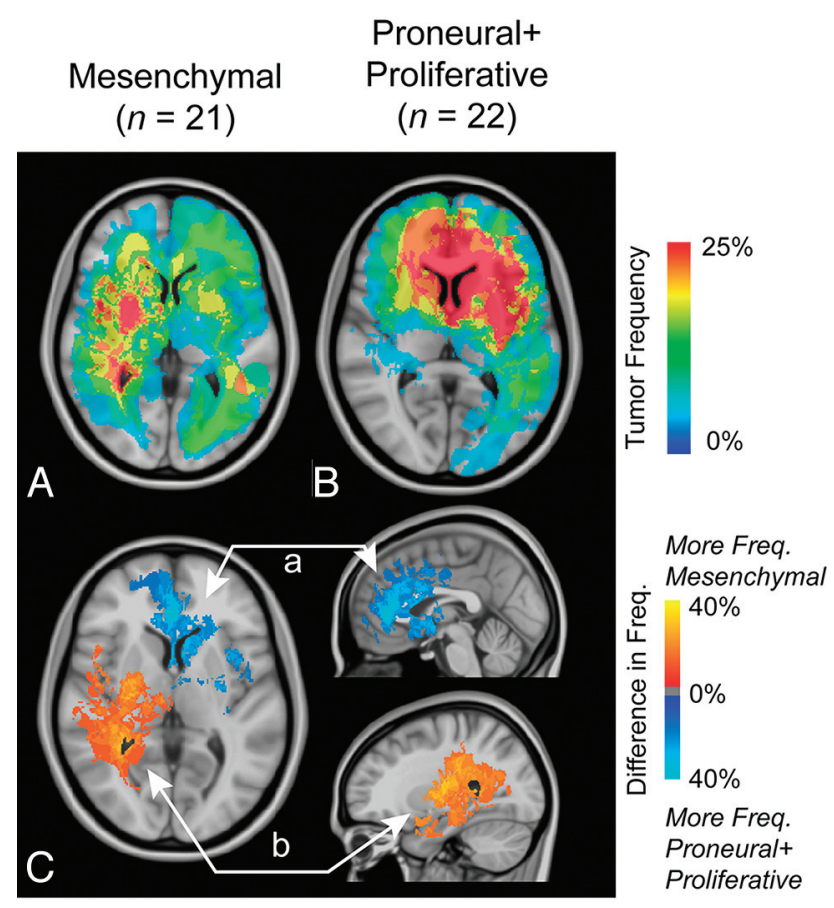

FIG 5. Atlas of gene expression subclassification. Frequency of T2/ FLAIR tumor occurrence in mesenchymal $(n=21)(A)$ and combined proneural and proliferative $(n=22)(B)$ phenotypes showing a similar pattern to the IDH1 mutant phenotypes. C, ADIFFI statistical analysis isolates 2 spatially distinct clusters, cluster a in the frontal lobe corresponding to tumors that are more frequently proneural or proliferative and cluster $b$ diffusely spread throughout the right hemisphere and surrounding the right posterior lateral ventricle. T2/FLAIR tumor frequency is illustrated.

\section{Overall Survival}

ADIFFI analysis identified 2 distinct clusters on separate hemispheres associated with either a short overall survival $(<12$ month overall survival; right hemisphere, a in Fig 9C) or a long overall survival ( $>36$-month overall survival; left hemisphere, $b$ in Fig 9C). Both T1 $+\mathrm{C}$ and T2/FLAIR tumor volumes were not significantly different among different overall survivals $(\mathrm{T} 1+\mathrm{C}$ and T2/FLAIR, 1-way ANOVA; $<12$-month overall survival, 1236-month overall survival, $>36$-month overall survival; $P>.05$ ).

\section{DISCUSSION}

The current study demonstrates the utility of a comprehensive atlas of stereotactic tumor locations composed of $>500$ de novo glioblastomas and 10 different demographic, -omic, and interventional phenotypes. Restricted patterns in the topographic distribution of glioblastomas appear to arise from specific phenotypes, which appear consistent with the hypothesis of distinct glioma cells of origin. Our results appear to support the hypothesis that glioblastomas may arise from neural stem cells near the subventricular zone $e^{2,3}$ and may migrate along periventricular white matter tracts.

\section{Atlas Stratifying the Extent of Resection Highlights Broca and Wernicke Areas}

The extent of surgical resection is a significant prognostic factor in glioblastoma, ${ }^{20}$ which also reflects surgical practices of the attending neurosurgeons. At our institution, $\mathrm{fMRI}$ is performed to spare eloquent function and limit morbidity; therefore, we expected a high frequency of tumors in language functional areas to occur more often in subtotal resection. Consistent with this hypothesis, results from the current study clearly outlined a cluster of tissue connecting Broca and Wernicke areas as the primary region occurring most frequently in subtotal resection compared with gross total resection.

\section{Atlas of MGMT Promoter Methylation Detects Temporal Lobe Asymmetry, Which May Be Dependent on Mutation of IDH1}

A recent report containing a subset of patients from the current study demonstrated, in patients with glioblastoma, significant lateralization of MGMT promoter methylated tumors to the left temporal lobe and lateralization of MGMT unmethylated tumors to the right hemisphere. ${ }^{10}$ These findings were also consistent with those in the current study with more patients. Hemispheric asymmetry in brain structures is well documented, ${ }^{21,22}$ particularly in the temporal lobe. ${ }^{23}$ Hemispheric differences in gene expression have also been identified, ${ }^{24}$ including the suppressor of the fused homolog gene in the sonic hedgehog pathway that regulates brain tumor proliferation. ${ }^{25}$ Most interesting, localization of MGMT promoter methylated tumors was also present when examining the interaction between $\mathrm{IDH} 1$ mutation status and MGMT promoter methylation, suggesting that a significant number of IDH1 mutant tumors are localized to the frontal lobe only in MGMT promoter methylated tumors. The topographic link between IDH1 mutation and MGMT promoter methylation status supports findings that these 2 phenotypes are tightly correlated. ${ }^{26}$

\section{Radiographic Atlases Identify a Subset of Tumors with Frontal Lobe Predominance}

Results from the current study suggest that glioblastomas occurring in young pa-
FIG 6. Atlases of PTEN loss and PTEN deficiency. A, ADIFFI statistical analysis identified a cluster in the left frontal lobe associated with a high frequency of glioblastomas lacking PTEN los (PTEN loss $[n=82]$ and lacking PTEN loss $[n=13])$. B. Additionally, a cluster was isolated in periventricular white matter regions near the posterior aspect of the left lateral ventricle that may be associated with a high frequency of glioblastomas with PTEN protein deficiency (PTEN deficient $[n=12]$ and PTEN intact $[n=16])$. T1 $+C$ tumor frequency is illustrated. 


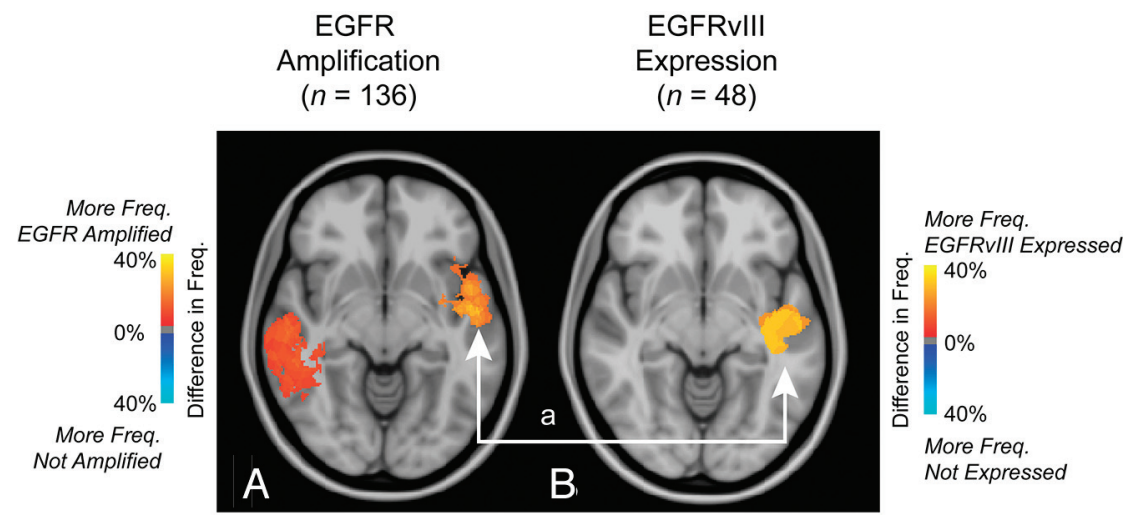

FIG 7. Atlases of EGFR amplification and EGFR variant 3 expression. A, ADIFFI analysis isolates 2 clusters associated with EGFR amplification (EGFR amplified $[n=79]$ and EGFR not amplified $[n=57])$. B, One cluster a was also identified by examining EGFR variant 3 expression (EGFR variant 3 expressed $[n=12]$; EGFR variant 3 not expressed $[n=36])$. $T 1+C$ tumor frequency is illustrated.

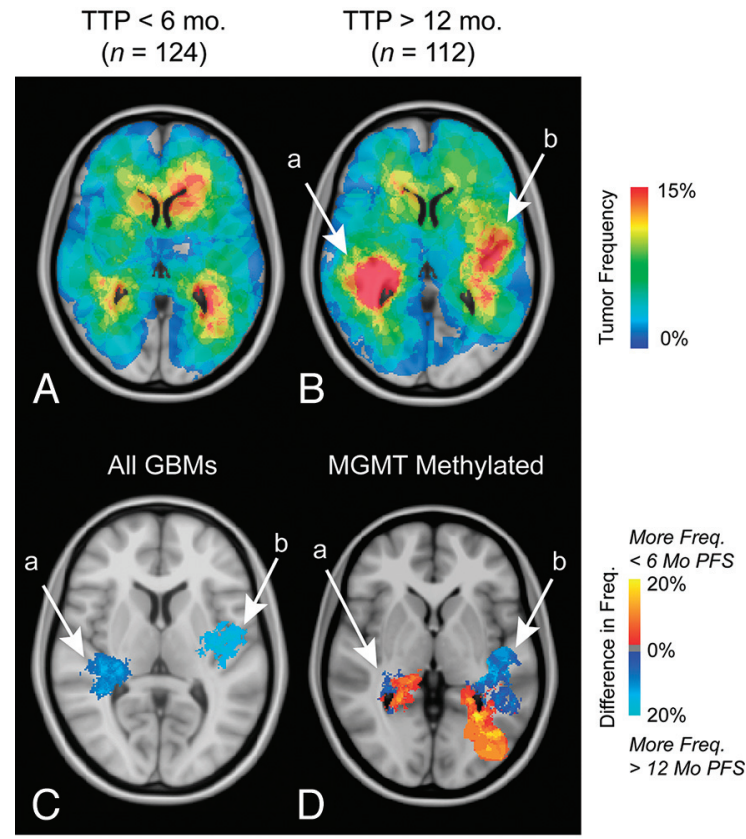

FIG 8. Atlas of the radiochemotherapy interventional phenotype. Frequency of $\mathrm{Tl}+\mathrm{C}$ tumor occurrence for nonresponders (time to progression $<6$ months, $n=124$ ) (A) and long-term responders (time to progression $>12$ months, $n=112)(B)$. C, ADIFFI results identify 2 distinct clusters, $a$ and $b$, associated with a high frequency of tumor occurrence in long-term responders. $D$, Within MGMT promoter methylated tumors, which are known to have a survival advantage, ADIFFI analysis identifies the same 2 clusters, but they contain a mixture of responders and nonresponders. $\mathrm{Tl}+\mathrm{C}$ tumor frequency is illustrated.

tients and harboring IDH1 mutation, having proneural and/or proliferative gene expression and a lack of a loss of PTEN, are more likely to be localized to the frontal lobe. A recent study has suggested that IDH1 mutant gliomas arise from a distinct neural precursor population that may be spatially restricted to the frontal lobe and temporally restricted to younger patients. ${ }^{7}$ This report also established a link between the proneural gene expression subgroup and IDH1 mutant tumors as well as the mesenchymal subgroup and IDH1 wild-type tumors, which the current study suggests are diffuse and preferentially in the right hemisphere. Lower frequencies of PTEN loss were also detected in IHD1 mutant compared with wild-type tumors in both studies.

\section{Radiographic Atlases Identify Regions Associated with Treatment Response and Survival}

Results from the current study suggest that the periventricular white matter regions in the right hemisphere were associated with a short overall survival from the time of diagnosis. Similarly, this region was also shown to be contiguous with regions possessing the mesenchymal gene expression profile, lack of MGMT promoter methylation, and IDH1 wildtype classification, all of which are known to have a poor prognosis. ${ }^{7}$ In the radiochemotherapeutic interventional phenotype and when stratified by overall survival, tumors in the left temporal lobe had the best prognosis. When examining the response to radiochemotherapy in only the MGMT promoter methylated tumors, a similar region in the left temporal lobe was associated with a favorable response.

Bihemispheric clusters identified for EGFR amplification may explain contradicting results as to the prognostic relevance of EGFR amplification. ${ }^{27}$ When one considers both EGFR amplification and EGFR variant 3 expression, a common cluster occurs in the left temporal lobe anterior to the region identified by MGMT promoter methylation. As previously mentioned, this particular region was associated with a statistically longer progression-free survival and overall survival, supporting the findings from other investigators as to the negative prognostic value of EGFR amplification. ${ }^{28}$ Nevertheless, the relationship between tumor response to therapy, survival, and molecular phenotypes likely involves complex interactions that still need to be further delineated. We hypothesize that radiographic atlases illustrating the spatial correlations among these different phenotypes, as presented in the current study, may be important for deconvolving these interactions.

\section{Limitations}

There are a number of critical limitations to the current study. Because of the retrospective nature of the current study, we were unable to standardize the imaging data, which included heterogeneity with respect to field strength, type of gadolinium chelate used, imaging-section thickness, and the type of T2-weighted sequence (T2 FSE versus FLAIR). Second, image registration of anatomically distorted brains into standard stereotactic atlas space can be challenging. Despite our best effort to align images manually (when necessary) and verification of alignment by 2 independent observers, this lack of accuracy is a potential limitation.

\section{CONCLUSIONS}

Radiographic atlases identifying the location and frequency of glioblastoma tumor occurrence for specific demographic, -omic, and interventional phenotypes can provide new insight into po- 


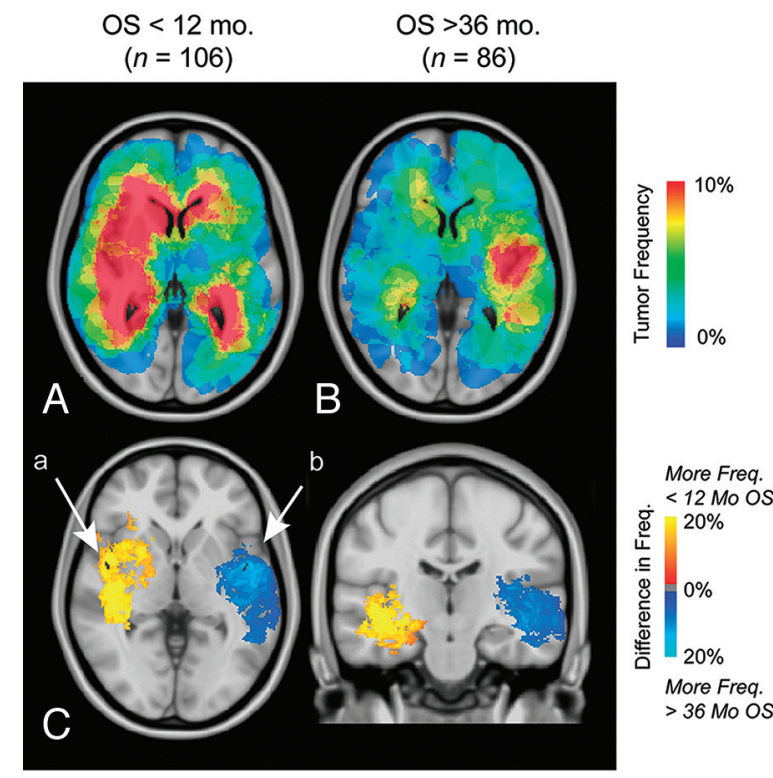

FIG 9. Atlas of overall survival. Frequency of $\mathrm{T} 1+\mathrm{C}$ tumor occurrence for short-term $(<12$ months, $n=106)(A)$ and long-term survivors (>36 months, $n=86$ ) (B). C, ADIFFI statistical analysis isolates 2 clusters in the temporal lobes, $a$ and $b$, associated with a high frequency of tumor occurrence in short-term and long-term survivors, respectively.

tential overlap between prognostic variables and may help identify niche locations for glioma cells of origin. Results from the current study suggest that tumor laterality (left versus right hemisphere) and frontal lobe involvement may play a role in the particular molecular and genetic profile of tumors and their response to cytotoxic treatment and overall survival. Future studies aimed at determining the possible biologic mechanisms for these findings are warranted.

\section{ACKNOWLEDGMENTS}

We thank Taryar Zaw, Kourosh Naeini, Nathan Stumpf, Yalda Behbahanian, and Sandy Mong for their tireless effort in segmenting tumor volumes and Heidi Phillips, MD, at Genentech, for her valuable guidance.

Disclosures: Albert Lai-RELATED: Consultancy: Genentech. William H. Yong—RELATED: Grant: National Institutes of Health, Ivy Foundation, UNRELATED: Grants/ Grants Pending: National Institutes of Health (possibly), ${ }^{*}$ Other: Amgen,* Genentech,* Tocagen,* Comments: collaborative research. Whitney B. PopeUNRELATED: Consultancy: Genentech, Roche. Harry V. Vinters-RELATED: Grant: National Institutes of Health, ${ }^{*}$ Rasmussen's Encephalitis Children's Project, ${ }^{\star}$ National Institute of Neurological Disorders and Stroke, ${ }^{*}$ California Pediatric Neuropathology Consortium, * Comments: I supervise the Neuropathology and "Blood and Tissue" Cores for several National Institutes of Health-funded projects, UNRELATED: Stock/ Stock Options: significant holdings in 3M, Teva Pharma, GE, Pfizer, Glaxo SmithKline Beecham, but none relevant to this work except that I suppose some imaging was done on GE machines. * Linda M. Liau—UNRELATED: Grants/Grants Pending: Northwest Biotherapeutics.* Paul S. Mischel—UNRELATED: Board Membership: James McDonnell Brain Cancer Advisory Board, Comments: They pay me $\$ 5000$ to review grants. I am also on multiple journal editorial boards and external advisory boards, without payment. They do pay my travel to external advisory board meetings, Payment for Lectures (including service on Speakers Bureaus): I have given multiple lectures at various university department symposia around the United States and at Keystone meetings. I sometimes receive honoraria, usually $\$ 500-\$ 1000$. Timothy F. Cloughesy_UNRELATED: Consultancy: Roche, Genentech, Novartis, Merck Serono, Merck, Payment for Lectures (including service on Speakers Bureaus): Merck. * Money paid to the institution.

\section{REFERENCES}

1. Simpson JR, Horton J, Scott C, et al. Influence of location and extent of surgical resection on survival of patients with glioblastoma multiforme: results of three consecutive Radiation Therapy Oncology Group (RTOG) clinical trials. Int J Radiat Oncol Biol Phys 1993; 26:239-44

2. Sanai N, Alvarez-Buylla A, Berger MS. Neural stem cells and the origin of gliomas. $N$ Engl J Med 2005;353:811-22

3. Wechsler-Reya R, Scott MP. The developmental biology of brain tumors. Annu Rev Neurosci 2001;24:385-428

4. Zlatescu MC, TehraniYazdi A, Sasaki H, et al. Tumor location and growth pattern correlate with genetic signature in oligodendroglial neoplasms. Cancer Res 2001;61:6713-15

5. Marino S, Vooijs M, van Der Gulden H, et al. Induction of medulloblastomas in p53-null mutant mice by somatic inactivation of $\mathrm{Rb}$ in the external granular layer cells of the cerebellum. Genes Dev 2000; 14:994-1004

6. Poppleton H, Gilbertson RJ. Stem cells of ependymoma. Br J Cancer 2007;96:6-10

7. Lai A, Kharbanda S, Tran A, et al. Evidence for sequenced molecular evolution of IDH1 mutant glioblastoma from a distinct cell of origin. J Clin Oncol 2011;29:4482-90

8. Zinn PO, Mahajan B, Sathyan P, et al. Radiogenomic mapping of edema/cellular invasion MRI-phenotypes in glioblastoma multiforme. PLoS One 2011;6:e25451

9. Pope WB, Chen JH, Dong J, et al. Relationship between gene expression and enhancement in glioblastoma multiforme: exploratory DNA microarray analysis. Radiology 2008;249:268-77

10. Ellingson BM, Cloughesy TF, Pope WB, et al. Anatomic localization of O6-methylguanine DNA methyltransferase (MGMT) promoter methylated and unmethylated tumors: a radiographic study in $\mathbf{3 5 8}$ de novo human glioblastomas. Neuroimage 2012;59:908-16

11. Carrillo JA, Lai A, Nghiemphu PL, et al. Relationship between tumor enhancement, edema, IDH1 mutational status, MGMT promoter methylation, and survival in glioblastoma. AJNR Am J Neuroradiol 2012;33:1349-55

12. Ellingson BM, Cloughesy TF, Lai A, et al. Quantitative volumetric analysis of conventional MRI response in recurrent glioblastoma treated with bevacizumab. Neuro Oncol 2011;13:401-09

13. Bullmore ET, Suckling J, Overmeyer S, et al. Global, voxel, and cluster tests, by theory and permutation, for a difference between two groups of structural MR images of the brain. IEEE Trans Med Imaging 1999;18:32-42

14. Hegi ME, Diserens AC, Gorlia T, et al. MGMT gene silencing and benefit from temozolomide in glioblastoma. N Engl J Med 2005;352: 997-1003

15. Lai A, Tran A, Nghiemphu PL, et al. Phase II study of bevacizumab plus temozolomide during and after radiation therapy for patients with newly diagnosed glioblastoma multiforme. J Clin Oncol 2011; 29:142-48

16. Phillips HS, Kharbanda S, Chen R, et al. Molecular subclasses of high-grade glioma predict prognosis, delineate a pattern of disease progression, and resemble stages in neurogenesis. Cancer Cell 2006; 9:157-73

17. Mellinghoff IK, Wang MY, Vivanco I, et al. Molecular determinants of the response of glioblastomas to EGFR kinase inhibitors. $N$ Engl J Med 2005;353:2012-24

18. Yoshimoto K, Dang J, Zhu S, et al. Development of a real-time RTPCR assay for detecting EGFRvIII in glioblastoma samples. Clin Cancer Res 2008;14:488-93

19. Stupp R, Mason WP, van den Bent MJ, et al. Radiotherapy plus concomitant and adjuvant temozolomide for glioblastoma. $N \mathrm{Engl}$ J Med 2005;352:987-96

20. Bauchet L, Mathieu-Daude H, Fabbro-Peray P, et al. Oncological patterns of care and outcome for 952 patients with newly diagnosed glioblastoma in 2004. Neuro Oncol 2010;12:725-35

21. Sun T, Walsh CA. Molecular approaches to brain asymmetry and handedness. Nat Rev Neurosci 2006; 7:655-62 
22. Toga AW, Thompson PM. Mapping brain asymmetry. Nat Rev Neurosci 2003;4:37-48

23. Rubens AB, Mahowald MW, Hutton JT. Asymmetry of the lateral (Sylvian) fissures in man. Neurology 1976;26:620-24

24. Sun T, Patoine C, Abu-Khalil A, et al. Early asymmetry of gene transcription in embryonic human left and right cerebral cortex. Science 2005;308:1794-98

25. Xu Q, Yuan X, Liu G, et al. Hedgehog signaling regulates brain tumor-initiating cell proliferation and portends shorter survival for patients with PTEN-coexpressing glioblastomas. Stem Cells 2008; 26:3018-26
26. Sanson M, Marie $\mathrm{Y}$, Paris $\mathrm{S}$, et al. Isocitrate dehydrogenase 1 codon 132 mutation is an important prognostic biomarker in gliomas. J Clin Oncol 2009;27:4150-54

27. Newcomb EW, Cohen H, Lee SR, et al. Survival of patients with glioblastoma multiforme is not influenced by altered expression of p16, p53, EGFR, MDM2 or Bcl-2 genes. Brain Pathol 1998;8: 655-67

28. Etienne MC, Formento JL, Lebrun-Frenay C, et al. Epidermal growth factor receptor and labeling index are independent prognostic factors in glial tumor outcome. Clin Cancer Res 1998;4: 2383-90 